DOI: 10.21915 /BIMAS.2019101

\title{
GENERALIZED TWISTED QUANTUM DOUBLES OF A FINITE GROUP AND RATIONAL ORBIFOLDS
}

\author{
GEOFFREY MASON ${ }^{1, a}$ AND SIU-HUNG NG ${ }^{2, b}$
}

Dedicated to Bob Griess on the occasion of his $71^{\text {st }}$ birthday.

\footnotetext{
${ }^{1}$ Department of Mathematics, UC Santa Cruz, CA 95064, USA.

${ }^{a}$ E-mail: gem@ucsc.edu

${ }^{2}$ Department of Mathematics, Louisiana State University, Baton Rouge, LA 70803, USA.

${ }^{b}$ E-mail: rng@math.lsu.edu
}

\begin{abstract}
In previous work the authors introduced a new class of modular quasi-Hopf algebra $D^{\omega}(G, A)$, associated to a finite group $G$, a central subgroup $A$ and a 3-cocycle $\omega \in Z^{3}\left(G, \mathbb{C}^{\times}\right)$. In the present paper we propose a description of the class of orbifold models of rational vertex operator algebras whose module category is tensor equivalent to $D^{\omega}(G, A)$-mod. The paper includes background on quasi-Hopf algebras and a discussion of some relevant orbifolds.
\end{abstract}

\section{Introduction}

Since its introduction by Dijkgraaf, Pasquier and Roche [4], the twisted quantum double has been a source of inspiration in the related areas of quasiHopf algebras, modular tensor categories (MTC), and orbifold models of holomorphic vertex operator algebras (VOA). It follows from the work [20] of Müger that the module category $D^{\omega}(G)$-mod of a twisted quantum double of a finite group $G$ (notation and further details are provided below) is a MTC. Even before the idea of a MTC existed, DPR had more-or-less

Received March 22, 2017 and in revised form December 3, 2017.

AMS Subject Classification: 16T99, 18D99, 17 B69.

Key words and phrases: Finite group, generalized twisted quantum double, modular tensor category.

The first author thanks the Simon Foundation, grant \#427007, for its support.

The second author was partially support by the NSF grant DMS 1501179. 
conjectured [4] that if $V$ is a holomorphic VOA admitting $G$ as a group of automorphisms then $V^{G}$-mod is equivalent to $D^{\omega}(G)$-mod for some 3-cocycle $\omega \in Z^{3}\left(G, \mathbb{C}^{\times}\right)$. Kirillov considered the conjecture from the perspective of fusion categories [14]. His work would imply the conjecture if one can show that all the $g$-twisted modules of $V$ form a $G$-graded fusion category. This condition amounts to the rationality of $V^{G}$. With recent advances, this conjecture is now known for a large family of groups $G$. The recent work [19] of Miyamoto and Carnahan proves that $V^{G}$ is rational for any solvable group $G$. A complete solution to the conjecture seems to be within reach.

On the other hand, much less is known in the case of rational orbifolds, i.e., orbifolds $V^{G}$ where $V$ is a rational $V O A$, but not necessarily holomorphic. Already in [4], the authors asked for a description of the $c=1 \mathrm{ADE}$ orbifolds $V^{G}$ where $V=L\left(s l_{2}, 1\right)$ is the affine algebra of level 1 associated to $s l_{2}$ (alternatively, the rank 1 lattice theory $V_{\sqrt{2} \mathbb{Z}}$ associated with the $A_{1}$ root lattice) and $G$ a finite subgroup of $S O(3, \mathbb{R})$. Until recently there was no really satisfactory answer to this question. Fusion rules and $S$ - and $T$-matrices for these theories have long been known (the icosahedral case proved to be particularly intractable) but a quasiHopf algebra replacing the twisted double was missing. More generally, there does not seem to be even a conjectural description of $V^{G}$-mod in the literature for any reasonably substantial class of rational orbifolds beyond the holomorphic case.

In our recent work [15] we introduced a generalization of the twisted quantum double, denoted by $D^{\omega}(G, A)$, which is a certain quasiHopf algebra quotient of $D^{\omega}(G)$ obtained from a central subgroup $A$ of $G$. (The case $A=1$ reduces to $D^{\omega}(G)$.) We gave necessary and sufficient conditions that $D^{\omega}(G, A)$-mod is a MTC. The purpose of the present paper is to present a conjectural description of those rational orbifolds $V^{G}$ whose module category is equivalent to some $D^{\omega}(G, A)$-mod, and to discuss a few examples. These include the ADE examples mentioned above, thus providing an answer to the question raised by DPR.

The paper is organized as follows. In Section 2 we present a general discussion of quasiHopf algebras, with emphasis on the construction and properties of $D^{\omega}(G, A)$. In Section 3 we state our conjecture relating $D^{\omega}(G, A)$ to certain rational orbifolds, and in Section 4 we consider some illustrative examples. 
We thank Terry Gannon for his interest and input, and for suggesting that this paper be written.

\section{QuasiHopf Algebras and $D^{\omega}(G, A)$}

In this Section we provide some background, taken from [15], about the quasiHopf algebras $D^{\omega}(G, A)$, which we call generalized twisted quantum doubles.

We use the following notation for a finite group $G$. $\widehat{G}=\operatorname{Hom}\left(G, \mathbb{C}^{\times}\right)$is the group of characters of $G, x^{g}=g^{-1} x g(x, g \in G)$ is right conjugation in $G$, the centralizer of $x$ in $G$ is $C_{G}(x):=\left\{g \in G \mid x^{g}=x\right\}, Z(G):=\cap_{x} C_{G}(x)$ is the center of $G$.

We take our base field to be the complex numbers $\mathbb{C}$. A quasiHopf algebra is a tuple $(H, \Delta, \epsilon, \phi, \alpha, \beta, S)$, where $H$ is a unital algebra and $\Delta: H \rightarrow H \otimes H$ an algebra morphism that is quasicoassociative in the sense that there is a map $\phi$ (the Drinfeld associator) making the following diagram commutative:

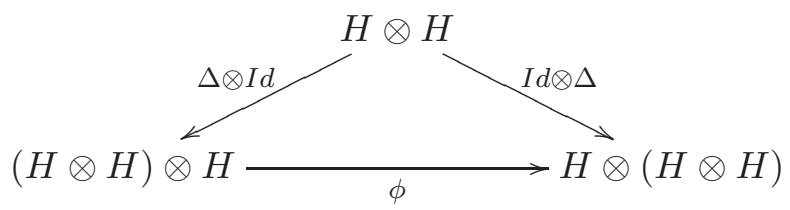

$S$ is the antipode, $\epsilon$ the counit, and $\alpha, \beta \in H$ are certain distinguished elements. We generally suppress all reference to these elements of a quasiHopf algebra; this will not impair the reader's understanding of what follows. One also requires $\phi$ to satisfy some cocycle conditions in the form of certain diagrams involving fourfold tensor products of $H$ that are required to commute. Again we will generally suppress such details. A Hopf algebra is a quasiHopf algebra for which $\alpha=\beta=1$ and $\phi=1 \otimes 1 \otimes 1$. For further background on quasiHopf algebras, see [10], [13] and [17].

One of the great virtues of a quasiHopf algebra $H$ is that the category $H$-mod of (finite-dimensional) $H$-modules is a finite tensor category, though not necessarily a MTC.

Fix a finite group $G$. The group algebra $\mathbb{C} G$ is a familiar example of a Hopf algebra, the coproduct being defined by $\Delta(g)=g \otimes g(g \in G)$. Dualizing, 
the dual group algebra $\mathbb{C}^{G}$ has basis $e_{g}(g \in G)$ dual to the basis of group elements in $G$. It is a Hopf algebra with product and coproduct defined by

$$
e_{g} e_{h}=\delta_{g, h} e_{g}, \quad \Delta: e_{g} \mapsto \sum_{a b=g} e_{a} \otimes e_{b}
$$

Now fix a normalized multiplicative 3-cocycle $\omega \in Z^{3}\left(G, \mathbb{C}^{\times}\right)$. A basic example of a quasiHopf algebra is the twisted dual group algebra $\mathbb{C}_{\omega}^{G}$, obtained from $\mathbb{C}^{G}$ just by replacing $1 \otimes 1 \otimes 1$ by a more interesting Drinfeld associator defined by multiplication by

$$
\phi:=\sum_{a, b, c} \omega(a, b, c)^{-1} e_{a} \otimes e_{b} \otimes e_{c} .
$$

Here, the cocycle conditions amount to the 3-cocycle identity satisfied by $\omega$.

The twisted quantum double $D^{\omega}(G)=\mathbb{C}_{\omega}^{G} \otimes \mathbb{C} G$ occurs as the middle term of a sequence of morphisms of quasiHopf algebras

$$
\mathbb{C}_{\omega}^{G} \stackrel{i}{\longrightarrow} D^{\omega}(G) \stackrel{p}{\longrightarrow} \mathbb{C} G
$$

where $i\left(e_{g}\right)=e_{g} \otimes 1, p\left(e_{g} \otimes x\right)=\delta_{g, 1} x . D^{\omega}(G)$ is itself a quasiHopf algebra with the following product and coproduct:

$$
\begin{aligned}
& \left(e_{g} \otimes x\right) \cdot\left(e_{h} \otimes y\right)=\theta_{g}(x, y) \delta_{g^{x}, h} e_{g} \otimes x y \\
& \Delta: e_{g} \otimes x \mapsto \sum_{a b=g} \gamma_{x}(a, b) e_{a} \otimes x \otimes e_{b} \otimes x
\end{aligned}
$$

The scalars $\theta_{g}(x, y), \gamma_{x}(a, b)$ are determined by $\omega$ as follows:

$$
\begin{aligned}
& \theta_{g}(x, y):=\frac{\omega(g, x, y) \omega\left(x, y, g^{x y}\right)}{\omega\left(x, g^{x}, y\right)}, \\
& \gamma_{x}(a, b):=\frac{\omega(a, b, x) \omega\left(x, a^{x}, b^{x}\right)}{\omega\left(a, x, b^{x}\right)} .
\end{aligned}
$$

We remark that the 2-cochains defined by the $\theta$ 's and $\gamma$ 's have subtle properties which govern much of the behaviour of $D^{\omega}(G)$ - for example, the fact that $D^{\omega}(G)$ really is a quasiHopf algebra, which is not obvious. As another example, if we restrict $x, y$ to $C_{G}(g)$ then the 2-cochains $\theta_{g}, \gamma_{g}$ 
coincide and become 2-cocycles. We informally record this as

$$
\gamma_{g}=\theta_{g} \in Z^{2}\left(C_{G}(g), \mathbb{C}^{\times}\right),
$$

where it is understood that the domains of $\gamma_{g}, \theta_{g}$ are here restricted to $C_{G}(g)$.

Since $D^{\omega}(G)$-mod is the Drinfeld center of $\operatorname{Vec}(G, \omega)$, the fusion category of $G$-graded vector space with the associativity given by $\omega$, it follows from [20] that $D^{\omega}(G)$-mod is a MTC.

We now fix another piece of data, namely a central subgroup $A \subseteq Z(G)$, and introduce

$$
D^{\omega}(G, A):=\mathbb{C}_{\omega}^{G} \otimes \mathbb{C}(G / A)
$$

Notice that because the conjugation action of $A$ on $G$ is trivial then the identical formulas used to define the operations in $D^{\omega}(G)$ still make sense in $D^{\omega}(G, A)$. When $\omega$ is compatible with $A$, one can equip $D^{\omega}(G, A)$ with a product and coproduct similar to the case of $D^{\omega}(G)$ so that $D^{\omega}(G, A)$ is a quasiHopf algebra.

Now it is natural to ask if, for suitable $\pi^{\prime}$, there is a commuting diagram of quasiHopf algebras and morphisms

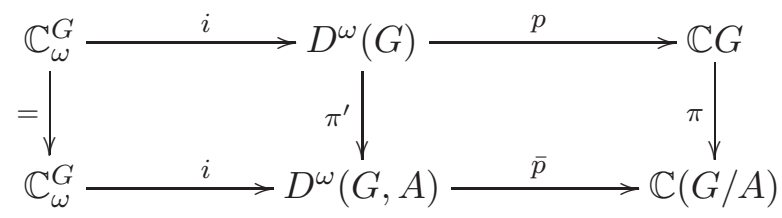

where $\pi(x)=x A$ and $\bar{p}\left(e_{g} \otimes x A\right)=\delta_{g, 1} x A$ for $x \in G$. In case $\pi^{\prime}$ exists, it will satisfy $\pi^{\prime}\left(e_{g} \otimes x\right)=\lambda e_{g} \otimes x A$ for a scalar $\lambda$ that depends on $g$ and $x$.

Generally there will be no such $\pi^{\prime}$, but we can give necessary and sufficient conditions for its existence. To explain this we first consider the grouplike elements of $D^{\omega}(G)$. These are the (nonzero) elements $u \in D^{\omega}(G)$ such that $\Delta(u)=u \otimes u$. As in the case of Hopf algebras, the group-like elements form a multiplicative subgroup $\Gamma^{\omega}(G) \subseteq D^{\omega}(G)^{\times}$of the group of units. We are more interested in the central group-like elements, which is the subgroup $\Gamma_{0}^{\omega}(G) \subseteq \Gamma^{\omega}(G)$ consisting of elements that commute with all elements 
of $D^{\omega}(G)$. One can show (cf. [17]) that there is a diagram of short exact sequences of groups (actually central extensions)

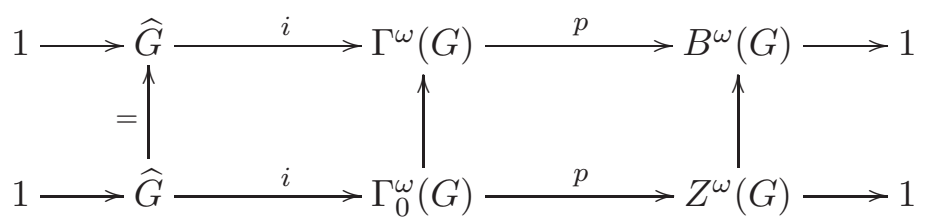

where

$$
B^{\omega}(G):=\left\{g \in G \mid \gamma_{g} \in B^{2}\left(G, \mathbb{C}^{\times}\right)\right\}, \quad Z^{\omega}(G):=B^{\omega}(G) \cap Z(G),
$$

and vertical arrows are containments.

We emphasize that here, unlike the situation of (2.3), in order for $g$ to lie in $B^{\omega}(G)$ it is necessary that the 2-cochain $\gamma_{g}$ be a 2-coboundary on $G$ rather than just the centralizer $C_{G}(g)$. On the other hand, if $g \in Z(G)$ then the context of (2.3) pertains, so that $\gamma_{g}=\theta_{g}$ is always a 2-cocycle on $G$, and the requirement to belong to $Z^{\omega}(G)$ is that this 2-cocycle is in fact a 2-coboundary.

It is shown in [15] that the existence of the morphism $\pi^{\prime}$ in (2.4) is equivalent to the existence of an enlarged diagram of central extensions

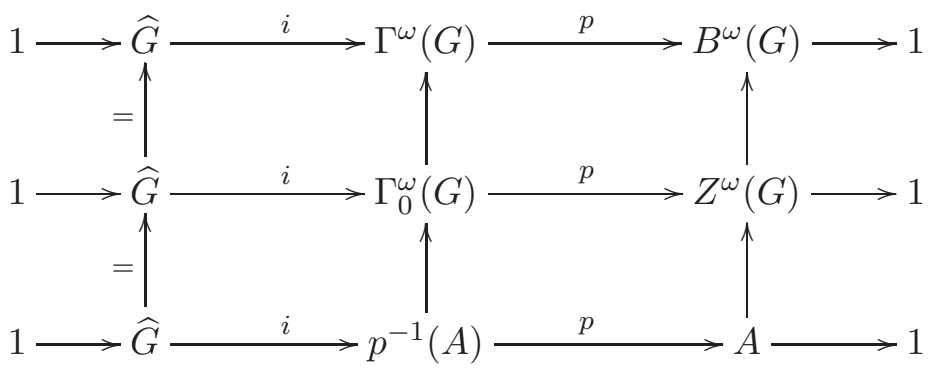

where vertical maps are again containments and the lower s.e.s splits.

What is being asserted here is the following: the central subgroup $A \subseteq$ $Z(G)$ is required to also lie in $B^{\omega}(G)$, i.e., the 2-cocycles $\theta_{g}$ for $g \in A$ are 2-coboundaries on $G$. Moreover, the s.e.s obtained by pulling-back $A$ along $p$ must split.

Once $\pi^{\prime}$ is available, it follows that $D^{\omega}(G, A)$-mod is a subcategory of $D^{\omega}(G)$-mod. However, Müger's theorem will generally not hold for $D^{\omega}(G, A)$, 
that is to say $D^{\omega}(G, A)$-mod is generally not a MTC. We will describe necessary and sufficient conditions, established in [15], that make this so.

First we say a bit more about the middle s.e.s in $(2.5)$. Given $g \in Z^{\omega}(G)$ we have $\gamma_{g}=\theta_{g} \in B^{2}\left(G, \mathbb{C}^{\times}\right)$, so that there is $\tau_{g} \in C^{1}\left(G, \mathbb{C}^{\times}\right)$satisfying $\delta \tau_{g}=\theta_{g}$, i.e.,

$$
\tau_{g}(x) \tau_{g}(y)=\theta_{g}(x, y) \tau_{g}(x y) \quad(x, y \in G)
$$

Because $\theta_{g}$ is a 2-coboundary, the twisted group algebra $\mathbb{C}^{\theta_{g}} G$ that it defines is isomorphic to the group algebra $\mathbb{C} G$, and $\tau_{g}$ defines a choice of isomorphism

$$
\mathbb{C}^{\theta_{g}} G \stackrel{\cong}{\longrightarrow} \mathbb{C} G, \quad x \mapsto \tau_{g}(x) x
$$

There is no canonical choice of $\tau_{g}$, but any two of them differ by a character $\chi \in \widehat{G}$. Indeed, we have

$$
\Gamma_{0}^{\omega}(G)=\left\{\sum_{x \in G} \tau_{g}(x) \chi(x) e_{x} \otimes g \mid \chi \in \widehat{G}, g \in Z^{\omega}(G)\right\} .
$$

A 2-cocycle $\beta \in Z^{2}\left(Z^{\omega}(G), \widehat{G}\right)$ that defines the central extension that is the middle s.e.s in (2.5) is given by the formula

$$
\beta(g, h)(k)=\frac{\tau_{g}(k) \tau_{h}(k)}{\tau_{g h}(k)} \theta_{k}(g, h) \quad\left(g, h \in Z^{\omega}(G), k \in G\right) .
$$

Because we are assuming that the lower s.e.s in (2.5) splits, the restriction of $\beta$ to $A$ is a 2-coboundary on $A$. That is, there is a 1-cochain $\nu \in C^{1}(A, \widehat{G})$ such that

$$
\beta(a, b)=\frac{\nu(a) \nu(b)}{\nu(a b)} \quad(a, b \in A) .
$$

Now we can show [15] that the formula

$$
(a \mid b)_{\nu}:=\frac{\tau_{a}(b) \tau_{b}(a)}{\nu(a)(b) \nu(b)(a)}
$$

defines a symmetric bicharacter $(\mid)_{\nu}: A \times A \rightarrow \mathbb{C}^{\times}$. Using results of Müger [20], [21], it follows that $D^{\omega}(G, A)$-mod is a MTC if, and only if, $(\mid)_{\nu}$ 
is nondegenerate. Actually, $(\mid)_{\nu}$ is the restriction of a natural bicharacter defined on $\Gamma_{0}^{\omega}(G)$, however we will not go into this here.

\section{Simple Current Orbifolds}

In the spirit of the proposal in [4] that the module categories of holomorphic orbifolds coincide with the module categories $D^{\omega}(G)$-mod, in this Section we describe those rational orbifolds $V^{G}$ expected to have the property that $V^{G}$-mod is equivalent to some $D^{\omega}(G, A)$. In this setting, the case $A=1$ will reduce to the holomorphic orbifold case of DPR.

We will generally be lapse about the detailed properties of the VOAs which we consider, but we will be concerned with rational VOAs $V$, which at the very least means that $V$ is a simple VOA and $V$-mod is a fusion category, i.e. a semisimple rigid tensor category with only finitely many simple objects. (See [7] for further background.) In fact we will only need a small subset of such theories, and to explain which ones these are we will review the theory of simple currents.

A simple current is a simple (or irreducible) $V$-module, call it $M$, with the property that for all simple $V$-modules $N$, the tensor product $M \otimes N$ is again a simple $V$-module. Otherwise stated, $M$ represents an element in the Grothendieck group of $V$-mod, and an object of Frobenius-Perron dimension one. Thanks to the associativity of $\otimes$, the distinct (isomorphism classes of) simple currents form a group with respect to tensor product of modules, called the group of simple currents. It is an abelian group because $V$-mod is also braided. The identity element is, of course, the vacuum space $V$. We are concerned here with rational VOAs $V$ with the property that every simple $V$-module is a simple current i.e., that $V$-mod is pointed. We call such a $V$ a simple current $V O A$. There are many examples of such theories. In addition to holomorphic VOAs, where $V$ is the only simple module, it is well-known that lattice theories $V_{L}$ associated to a positive-definite even lattice $L$ are also simple current VOAs. In this case, the group of simple currents is isomorphic to $L^{*} / L$ where $L^{*}$ is the dual lattice of $L$ [5].

Given a simple current VOA $V$, we may form the sum of all simple $V$-modules $M$ to obtain a larger space

$$
\widetilde{V}:=\oplus_{M} M
$$


$\widetilde{V}$ can be equipped with the structure of an abelian intertwining algebra in the sense of Dong-Leowsky [6]. See [8] for further details.

Conjecture. Suppose that $V$ is a simple current VOA with group of simple currents $A$. Let $F \subseteq \operatorname{Aut}(V)$ be a finite group of automorphisms of $V$ (the orbifold group) such that $F$ leaves invariant every simple $V$-module. Then there is central extension

$$
1 \longrightarrow A \longrightarrow G \longrightarrow F \longrightarrow 1
$$

and a 3-cocycle $\omega \in Z^{3}\left(G, \mathbb{C}^{\times}\right)$such that $V^{F}$-mod is equivalent to $D^{\omega}(G, A)$ mod as modular tensor categories. Conversely, if $D^{\omega}(G, A)$ exists and $D^{\omega}(G, A)$-mod is a MTC, then there is a simple current VOA $V$ with group of simple currents $A$ and a group of automorphisms $F=G / A$ such that $V^{F}$ mod is tensor equivalent to $D^{\omega}(G, A)$-mod.

\section{Examples}

We illustrate the Conjecture by discussing some examples in greater detail. Let the notation be as before.

Example 1. The holomorphic case.

Here, $A=1$ means that $V$ is a holomorphic VOA and $D^{\omega}(G, A)=D^{\omega}(G)$. The Conjecture thus reduces that of DPR.

Example 2. The case $F=1$, i.e., $G=A$ and $D^{\omega}(G, A)=\mathbb{C}_{\omega}^{G}$ as quasiHopf algebras.

We saw before that in order for $\pi^{\prime}$ to exist (i.e., $D^{\omega}(G, A)$ is a quasiHopf algebra quotient of $\left.D^{\omega}(G)\right)$ it is necessary that $A \subseteq Z^{\omega}(G)$, meaning that each $\theta_{g} \in B^{2}\left(G, \mathbb{C}^{\times}\right)$is a 2-coboundary for all $g \in A$. That is, $\omega \in Z^{3}\left(A, \mathbb{C}^{\times}\right)_{a b}$ is an abelian 3-cocycle on $A$ in the sense of [17], where such cocycles are studied extensively. They are closely related to the abelian cohomology groups introduced by Eilenberg and Maclane [11].

Thus in the case at hand, the Conjecture asserts that $V$-mod is tensor equivalent to $\mathbb{C}_{\omega}^{A}$ for an abelian 3-cocycle $\omega$ that is nondegenerate in a suitable sense. Rather than explain what degeneracy means here, we consider a special case in more detail. 
Let $L$ be an even lattice with bilinear form $\langle\rangle:, L \times L \rightarrow \mathbb{Z}$, and let $V_{L}$ be the corresponding lattice VOA. Then $A=L^{*} / L$. The conjecture says that $V_{L^{-}}$mod is tensor equivalent to the dual group algebra of $L^{*} / L$ twisted by an abelian 3-cocycle $\omega \in Z^{3}\left(L^{*} / L, \mathbb{C}^{\times}\right)$. The origin of $\omega$ is well-known in this case (cf. [6], Chapter 12 and [17], Section 11). Let $s: A \rightarrow L^{*}$ be a normalized section of the canonical s.e.s

$$
1 \longrightarrow L \longrightarrow L^{*} \longrightarrow A \longrightarrow 1
$$

Let $c_{0}: L^{*} \times L^{*} \rightarrow \mathbb{C}^{\times}$be an alternating bicharacter with $c_{0}(\alpha, \beta)=(-1)^{\langle\alpha, \beta\rangle}$ $(\alpha, \beta \in L)$. Set

$$
\omega(a, b, c)=(-1)^{\langle s(c), s(a)+s(b)-s(a+b)\rangle} c_{0}(s(c), s(a)+s(b)-s(a+b)) .
$$

$\omega$ is indeed an abelian 3-cocycle (cf. [17], Proposition 11.1) whose cohomology class is independent of the choice of bicharacter and section.

Example 3. The case $|A|=2$.

Here, we are discussing simple VOAs $V$ with just two simple modules. One of them is the adjoint module $V$, the other we denote by $M$. Roughly speaking, we may think of $\widetilde{V}=V \oplus M$ as a holomorphic super VOA, though this may not conform to some definitions. (This will not matter in the following discussion.)

Next we discuss results of [18] concerning the existence of $D^{\omega}(G, A)$ in the special case that $G$ is a finite group with exactly one subgroup of order 2. We take $A$ to be the unique subgroup of order 2. Let $T$ be a 2 Sylow subgroup of $G$. It is well-known that $T$ is either cyclic or generalized quaternion. Groups with a unique involution have 2-periodic cohomology by the Artin-Tate theory [1], and for such groups the 2-Sylow subgroup of $H^{3}\left(G, \mathbb{C}^{\times}\right)$is cyclic of order $|T|$. We call a 3 -cocycle $\omega \in Z^{3}\left(G, \mathbb{C}^{\times}\right)$a 2 generator if the corresponding cohomology class $[\omega] \in H^{3}\left(G, \mathbb{C}^{\times}\right)$has order divisible by $|T|$.

In the setting of the previous paragraph, we can prove [18] that $\pi^{\prime}$ always exists, so that $D^{\omega}(G, A)$ is a quasiHopf algebra quotient of $D^{\omega}(G)$. Moreover, $D^{\omega}(G, A)$-mod is a MTC if, and only if, $\omega$ is a 2 -generator. 
There are a number of interesting classes of finite groups with a unique subgroup $A$ of order 2 . The following lists some of them.

$$
\begin{aligned}
& S L_{2}(q) \quad(q \geq 3 \text { an odd prime power }) \\
& \text { 2. } A_{6}, 2 . A_{7}, 6 . A_{6}, 6 \cdot A_{7} \\
& \text { binary polyhedral groups }=\text { finite subgroups of } S U_{2}(\mathbb{C})
\end{aligned}
$$

Here, $S L_{2}(q)$ is the group of $2 \times 2$ matrices of determinant 1 over the finite field of cardinality $q$, and $2 . A_{n}$ is the 2 -fold perfect central extension of the alternating group $A_{n}$. For $n=6,7$ Schur discovered that there are exceptional 6 -fold perfect central extensions of $A_{n}$. There are a few overlaps among these groups: $S L_{2}(3)$ and $S L_{2}(5)$ are the binary octahedral and icosahedral groups respectively, and $2 . A_{6} \cong S L_{2}(9)$.

Our Conjecture says that for each pair $(G, \omega)$ such that $G$ has a unique subgroup of order 2 and $[\omega]$ is a 2-generator of $H^{3}\left(G, \mathbb{C}^{\times}\right)$, there is a holomorphic super VOA $\widetilde{V}=V \oplus M$ (in the sense described before) such that $G \subseteq \operatorname{Aut}(\widetilde{V}), G / A \subseteq \operatorname{Aut}(V)$ and $V^{G / A}$-mod is tensor equivalent to $D^{\omega}(G, A)$ mod.

As far as we know, the existence of a $\widetilde{V}$ for most groups $G$ on the list (4.1) is unknown. However, it is well-known that the binary polyhedral groups, and indeed $S U_{2}(\mathbb{C})$ itself, act on the $c=1$ holomorphic super VOA defined by the $A_{1}$ root lattice theory $V_{\sqrt{2} \mathbb{Z}}$, also known as the affine algebra $L_{s l_{2}, 1}$ of level 1 associated to the Lie algebra $s l_{2}(\mathbb{C})$. We review some of the details.

Adopting the lattice perspective, the irreducible modules for $V_{\sqrt{2} \mathbb{Z}}$ consist of the adjoint module $V_{\sqrt{2} \mathbb{Z}}$ and the module $M=V_{1 / \sqrt{2}+\sqrt{2} \mathbb{Z}}$ corresponding to the two cosets of $\sqrt{2} \mathbb{Z}$ in its dual lattice $\frac{1}{\sqrt{2}} \mathbb{Z}$. Thus $\widetilde{V}=V_{\sqrt{2} \mathbb{Z}} \oplus V_{1 / \sqrt{2}+\sqrt{2} \mathbb{Z}}$ $=V_{\frac{1}{\sqrt{2}}} \mathbb{Z}$. We have $\operatorname{Aut}(V)=S O_{3}(\mathbb{R})$, obtained by exponentiating the weight 1 states of $V$ (which form the Lie algebra $s l_{2}$ ). The projective action of this group on $M$ lifts to a linear action of its universal cover $S U_{2}(\mathbb{C})$, which is the full automorphism group of $\widetilde{V}$.

The binary polyhedral groups $G$ of even order are the finite subgroups of $S U_{2}(\mathbb{C})$ that contain the center $\{ \pm 1\}$. Setting $A=\{ \pm 1\}$, the preceding discussion shows that $G \subseteq \operatorname{Aut}(\widetilde{V})$ and $G / A \subseteq \operatorname{Aut}(V)$, so we have rational orbifolds $V^{G / A}$. These theories have been studied extensively in both the 
physical and mathematical literature, and the $S$ - and $T$-matrices are known. See, for example, [2] and [3] for type D. Dong and Nagatomo [9] computed the fusion rules for $V^{G / A}$ on the basis of the VOA axioms in all cases except the icosahedral example.

Now we can compute the $S$ - and $T$-matrices for all $D^{\omega}(G, A)$ whenever $G$ is a binary polyhedral group and $\omega$ is a 2-generator. Note that $H^{3}\left(G, \mathbb{C}^{\times}\right)$ is cyclic of order $|G|$ in this case, so that the number of 2-generators is exactly $|G| / 2$. Consider, for example the icosahedral group $G=S L_{2}(5)$. This is a group of order 120 , so there are 60 2-generators [ $\omega]$ of order $8,24,40$ or 120 . In the case of $V^{G / A}$-mod discussed above, the $S$-matrix appears to correspond to an $[\omega]$ of maximal order 120 .

In this way we get a large number of MTCs and accompanying modular data, and the Conjecture says that among them we should find the categories $V^{G / A}$-mod. This appears to indeed be the case, since in particular we can find matching $S$-matrices in all cases.

\section{References}

1. H. Cartan and S. Eilenberg, Homological Algebra, Seventh Ed., Princeton Landmarks in Mathematics, PUP, Princeton NJ, 1973.

2. M. Cuntz and C. Goff, An isomorphism between the fusion algebras of type $V_{L}^{+}$and type $D^{(1)}$ level 2, arxiv 0809.5186.

3. R. Dijkgraaf and E. Verlinde, Modular invariance and the Fusion Algebra, Nucl. Phys. $B$ (Proc. Suppl.) 5B (1988), 87-97.

4. R. Dijkgraaf, V. Pasquier and P. Roche, Quasi-Hopf algebras, group cohomology and orbifold models, in Integrable Systems and Quantum groups, World Scientific Publishing, NJ, 75-98.

5. C. Dong, Vertex algebras associated with even lattices, J. Alg., 160 (1993), 245-265.

6. C. Dong and J. Lepowsky, Generalized Vertex Algebras and Relative Vertex Operators, Birkhäuser, Boston, 1993.

7. C. Dong, H. Li and G. Mason, Twisted representations of vertex operator algebras, Math. Ann., 310(1998), 571-600.

8. C. Dong, H. Li and G. Mason, Simple currents and extensions of vertex operator algebras, CMP, 180 (1996), 671-707.

9. C. Dong and K. Nagatomo, Representations of the vertex operator algebra $V_{L}^{+}$for rank one lattice $L, C M P, 202$ (1999), 169-195.

10. V. Drinfeld, Quasi-Hopf algebras, Leningrad Math. J., 1 (1990), 1419-1457. 
11. S. Eilenberg and S. MacLane, Cohomology theory of abelian groups and homotopy theory I, Proc. Nat. Acad. Sci. USA, 36 (1950), 443-447.

12. D. Gorenstein, Finite Groups, Harper and Row, New York, 1968.

13. C. Kassel, Quantum Groups, Springer, New York, 1995.

14. A. Kirillov, Modular categories and orbifold models, Comm. Math. Phys., 229 (2002), no. 2, 309-335.

15. G. Mason and S.-H. Ng, Cleft extensions and quotients of twisted quantum doubles, in Developments in Mathematics 38, Developments and Retrospectives in Lie Theory (Algebraic Methods), Springer, New York, 2014, 229-246.

16. G. Mason and S.-H. Ng, Central invariants and Frobenius-Schur indicators for semisimple quasi-Hopf algebras, Adv. Math., 190 (2005), No. 1, 161-195.

17. G. Mason and S.-H. Ng, Group cohomology and gauge equivalence of some twisted quantum doubles, Trans. Amer. Math. Soc., 353 (2001), no. 9, 3465-3509.

18. G. Mason and S.-H. Ng, A new class of quasi-Hopf algebras and groups with one involution, to appear.

19. S. Carnahan and M. Miyamoto, Rationality of fixed-point vertex operator algebras, arXiv:1603.05645.

20. M. Müger, From subfactors to categories and topology II. The quantum double of tensor categories and subfactors, J. Pure Appl. Algebra, 180 No. 1-2 (2003), 159-219.

21. M. Müger, On the structure of modular categories, Proc. Lond. Math. Soc. (3), 87 (2003), 291-308. 